\title{
An Outlook on Nanocarriers to Improve Drug Efficacy of Chloroquine against SARS-CoV-2
}

\author{
Manash Jyoti Kashyap", Runjun Sarma ${ }^{2, *}$ \\ ${ }^{1}$ Technology Consultant, Bapuji Nagar, Bohotia Gaon Path, Jorhat - 785 006, Assam, India. \\ ${ }^{2}$ Formerly Assistant Professor, Mehr Chand Mahajan DAV College for Women, Sector 36-A, Chandigarh - 160 036, India.
}

\section{ART IC LE DETAILS}

Article history:

Received 28 June 2020

Accepted 18 July 2020

Available online 31 July 2020

Keywords:

SARS-CoV-2

COVID-19

QTc Prolongation

Nanotechnology

Drug Delivery

\begin{abstract}
A B S T R A C T
The sudden emergence of SARS-CoV-2 at the end of last year and its subsequent attainment of pandemic proportions have not only paralyzed the healthcare system but also destabilized the world economy due to subdued human activity in the past few months. Scientists the world over have been fervently working on finding a cure for the viral infection. Chloroquine and hydroxychloroquine are amongst the most popular medicines that are being considered as a counter to the SARS-CoV-2. However, the clinical trials of the drug both as a preventive for frontline healthcare professionals and as a cure for the infected have yielded mixed results. This is mainly due to the cardiotoxicity induced by the drug via QT prolongation when administered in high dosages. The prescription of the drug is further inadvisable if patients have been prescribed other QT prolonging drugs like azithromycin or have a known cardiac history. The authors have extensively studied literature on chloroquine mechanism of action and the successful use of nanocarriers as drug delivery vehicles against different viruses, malarial infections and in cancer therapy. The combination of nano-carrier and chloroquine/hydroxychloroquine is expected to overcome the limitations of the use of free chloroquine/hydroxychloroquine via increased drug efficacy and thus decreased chances of lethality arising from cardiotoxicity.
\end{abstract}

\section{Introduction}

An outbreak of a mysterious pneumonia emerged in a local seafood market of Wuhan city, in the Hubei province of China on December 2019 have jeopardized the global healthcare infrastructure till date [1]. This pneumonia like disease previously known as Novel coronavirus (2019$\mathrm{nCoV}$ ), have been officially renamed "Severe Acute Respiratory Syndrome Coronavirus 2 (SARS-CoV-2)" by the International Committee on Taxonomy of Viruses (ICTV) and as "COVID-19" by World Health Organization (WHO) on 11th February 2020. Thereafter, due to the rapid spread of the disease worldwide, the World Health Organization (WHO) declared it a pandemic on 11th March, 2020 [2]. The pathogen causing the infection was categorized into the Coronaviridae family using electronmicroscopical investigation of the isolated tissue cultures from the virion infected samples [3]. The whole-genome sequencing and the genetic analysis studies have revealed that SARS-CoV-2 is genetically related to SARS-CoV of the 2003 outbreak [4]. This infection has progressed in a highly destructive manner with uncontrolled proliferation amongst the world populace irrespective of climate, race and age groups. In this situation, it has become a challenge to produce effective and safe therapeutic drug(s) with known pharmacokinetics and optimal dosage, which can be used to treat or prevent SARS-CoV-2 infections. In this context, an innovative way of treating COVID-19 can be by the possible repositioning of drugs such as Interferon, Lopinavir/Ritonavir, Chloroquine Phosphate, Ribavirin, and Arbidol [5]. We will discuss here about the most frequently used drugs all over the world including chloroquine (CQ) and hydroxychloroquine (HCQ).

Chloroquine was discovered in 1934 and has been used to treat malaria since the late 1940s. Moreover, this antimalarial drug is shown to be effective in-vitro in extensive use of virus disease therapy after many clinical trials [6-8]. In March 30, 2020 the antimalarial drug, CQ $\left(\mathrm{C}_{18} \mathrm{H}_{26} \mathrm{ClN}_{3}\right)$ and its derivative HCQ $\left(\mathrm{C}_{18} \mathrm{H}_{26} \mathrm{ClN}_{3} \mathrm{O}\right)$ have been identified as an emergency treatment for COVID-19 by the US Food and Drug Administration (FDA) [5] though, they have many associated side effects arising from excessive doses and long-term use. CQ/HCQ has multiple functions, including alkalinisation of phagolysosome which results in suppressing the low-pH-dependent stages of viral replication, including fusion and un-coating [8].

The interaction of nanoparticles with microorganisms is fastrevolutionizing the biomedical field by offering advantages in both diagnostic and therapeutic applications including drug delivery $[9,10]$. This is because of the unique physico-chemical properties such as size, large surface to volume ratio and improved surface reactivity of the nanoparticles. The size of SARS-CoV-2 falls within the same size range $(60-140 \mathrm{~nm})$ as well as shape (spherical) [11] as the commonly studied synthetic nanoparticles. Therefore, research on nanoparticle conjugated Chloroquine can give an illuminating idea on the cellular uptake of the drug and its induced alterations in the SARS-CoV-2 [11].

\section{Action of Chloroquine (CQ)/Hydroxychloroquine (HCQ)}

Because of the various interesting biochemical effects of CQ and its derivative HCQ, they are potentially used as an antiviral drug apart from being used as an antimalarial agent. However, to reduce the effect of the novel SARS-CoV-2, it is very important to know the chemical behavior of CQ and HCQ and their precise mechanism of action at the cellular level.

Inhibition of biosynthesis of sialic acid is an important biochemical action shown by CQ which interferes with the pre entry step of the virion particle in to the host cell. The sialic acids are acidic monosaccharides found at the terminal position of the of sugar chains attached to the cell surface and cell transmembrane proteins. They play critical roles in cellular and molecular recognition [12]. However, the biosynthesis of sialic acid is a complex process. A nucleotide sugar, UDP-N-acetylglucosamine (UDP-GlcNAc), in particular UDP-GlcNAc 2-epimerase enzyme is the starting compound involved in the initial biosynthesis process of the sialic acid [13].

In case of SARS-CoV-2, sialic acid is attached at the end of the structural protein, spike (S) protein. S protein is one the most important structural proteins of SARS-CoV-2. Other important structural proteins of the virus include membrane (M) protein, envelope (E) protein, and the nucleocapsid $(\mathrm{N})$ protein. $S$ protein plays an important role in the entry of the virion into the host cell and participates in the cell attachment and host (cell) membrane fusion process [14]. The S protein of (SARS-CoV-2) uses angiotensin-converting enzyme 2 (ACE2) found in the cell membranes of the lungs, arteries, heart, kidneys and intestines, as the cellular receptor for the attachment of the virus [14-16]. CQ acts by inhibiting the enzyme 
quinone reductase 2 (a structural neighbour of UDP-N-acetylglucosamine 2-epimerase) and prevents recognition with the compatible sialic acid on the host cell. Thus, CQ can efficiently reduce the possibility of infection and spread of the virus $[17,18]$.

Both CQ and HCQ are amphiphilic diprotic weak bases and have protonated and unprotonated parts [19]. However, only the unprotonated part is capable of crossing the plasma membrane. Acid organelles like endosomes, Golgi vesicles and lysosomes are the sites of cellular action of CQ. CQ can diffuse freely and quickly across the membranes of cells and to these organelles [20]. Diffusion of CQ into the organelles is driven by their low $\mathrm{pH}$ due to the inverse proportionality between CQ diffusion and the $\mathrm{pH}$ value of the organelle environment. Utilization of $\mathrm{H}^{+}$ions of the lysosome towards protonation of the CQ causes a resultant increase in the $\mathrm{pH}$ of the vesicular environment. The protonated CQ is bound to the inside of the lysosome and is unable to diffuse out [21]. The lysosomal $\mathrm{H}^{+}$-ATPase act as proton pumps and helps in maintaining the acidic $\mathrm{pH}(\sim 5)$ in the lysosomal environment. These proton pumps function to replenish the supply of $\mathrm{H}^{+}$ions exhausted due to protonation of the CQ. Diffusion of CQ into the lysosome is driven by the continuous availability of protons by the $\mathrm{H}^{+}$-ATPase, thus irreversibly increasing the CQ concentration of the cell. This leads to elevation of the cell $\mathrm{pH}$ levels. Thus, the alteration of $\mathrm{pH}$ due to the influence of CQ/HCQ leads to dysfunction of enzymatic activity supporting post translational modification of the newly synthesized proteins of the virus. This inhibits the entry of the virus into the lysosome and also exosome release of virus particle from the infected host cell [22]. Moreover, CQ shows immunomodulatory effects by suppressing the secretion of cytokines, such as IL-6. These two factors mediate deregulation of the local inflammatory responses of human body and is primarily responsible for the SARS-CoV-2 associated mortality [23,24].

\section{Nanotechnology in Viral Infection Treatment}

Till now nanotechnology has been used in detection and drug delivery applications for the treatment of various diseases caused by human immunodeficiency virus (HIV), herpes simplex viruses (HSV), hepatitis B virus (HBV), influenza A virus (H1N1), cytomegalovirus (CMV), NIPAH virus etc. Different nano- platforms such as, nanoparticles [25], nanorods [26], lyposomes [27], dendrimers [28], micelles [29], nanosuspensions [30], lipid nanoparticles [31] etc. have been used as carriers of drugs used to treat viral infections. Nanoparticles can improve the activity of antiviral drugs by facilitating controlled release kinetics, enhancing bioavailability, modifying pharmacokinetics, reducing side effects and specially enabling the capacity of the drug to target specific sites [32-34].

\section{Prospect of CQ and Nanoparticle Composite on SARS-CoV-2 Treatment}

\subsection{Increased Drug Efficiency of CQ in Treatment}

Chloroquine (CQ) and its derivatives, commonly used as the first line of treatment of malarial infections are hydrophilic in nature. In addition to the extensive use of CQ against malarial infections, this drug and its derivatives have also been used against other diseases such as cancer, rheumatoid arthritis etc. $[35,36]$. Due to the recent outbreak of the SARSCoV-2, CQ is being widely investigated and in some cases prescribed as a potential drug against the viral infection [37].

Programmed release of the anti-viral drugs can provide a quantum leap towards controlling the spread of the SARS-CoV-2 in the host cell environments. It has been noticed that several anti-malarial drugs including CQ have been used with novel drug carriers based mostly on polymeric micro and nano drug delivery systems with sub-micron size range of 20-250 $\mathrm{nm}$ [38]. Embedding of CQ and other drugs for treatment of SARS-CoV-2 infections within nano-carriers provide benefits of sustained stability of the drug-delivery system against biological fluids up to availability of the drug at infected intracellular locations. These nanocarriers can be either inorganic or organic in nature. The inorganic nanoparticles frequently used are mainly those of noble metals, quantum dots, mesoporous silica and other silicon-based materials [39]. Organic nano-carriers for drugs are mostly based on biodegradable polymers, proteins, liposomes etc. Characteristics of the nano-carrier to be selected as a drug delivery vehicle include biological compatibility, non-toxic nature, affinity for the selected drug, facilitation of controlled diffusion of the drug at the infected area sometimes through a selectively permeable membrane or hydrophilic/ hydrophobic layer, adequate levels of elasticity and surface tension properties, easy metabolism in human body, optimized rate of degradation of the nano-carriers, selectively responsive to external stimuli like $\mathrm{pH}$ and temperature etc. Most polymer-based nanocarriers used for drug delivery systems are completely eliminated from the human body after fulfillment of the drug delivery objectives [40]. https://doi.org/10.30799/jnst.304.20060205
The mode of drug delivery by nano-carriers can be based on either passive or self-delivery models. The passive model of drug delivery is based on the use of hydrophobic nanoscale carrier, while, the self-delivery mechanism depends upon the direct conjugation of the drug such as CQ onto the nanostructured material [41]. The self-delivery model can be designed by incorporating nanostructures onto the drug. These nanostructures are stimulated by $\mathrm{pH}$, temperature, redox, magnetic and other types of environments at the intended intracellular locations affected by viral infection [38].

Hydrophilic behavior of CQ may interfere in its effective delivery to the infected areas if used in the free form i.e. without nano-carriers. It is reported that CQ have been used with a nano-carrier based on a hydrophobic and biodegradable polymer, PLA (poly-lactic acid) for the treatment of herpes simplex virus type 1 (HSV-1). The use of PLA based nano-drug delivery system in combination with CQ has helped overcome the limitation of the drug towards efficient intracellular delivery at the infected tissues thus avoiding multiple administration of the drug. This prevents the drug associated toxicity in the human body. The antiviral activity of the Chloroquine-nanoparticle (CQ-NP) conjugates significantly improved compared to free CQ. Greater uptake of the CQ by the virus cells have been observed when it was encapsulated with the nanoparticle (NP), providing slower release rate of the drug and higher cytotoxicity into the virus cells. For killing $50 \%$ virus cells the average concentration of free CQ $\left(222.6 \mu \mathrm{gmL}^{-1}\right)$ was found to be much higher than the CQ $\left(67.9 \mu \mathrm{gmL}^{-1}\right)$ in CQ-NP conjugates. The concentration that inhibited viral replication by $50 \%$ was found to be $6.7 \mu \mathrm{gmL}^{-1}$ and $4.3 \mu \mathrm{gmL}^{-1}$ for CQ and CQ-NP respectively. The values for the $100 \%$ inhibition of viral replication were $30 \mu \mathrm{gmL}^{-1}$ and $10 \mu \mathrm{gmL}^{-1}$. Though, with PLA encapsulation, the efficiency of CQ drug against HSV-1 improved in terms of reduced dosage of drug and controlled drug release, it was a challenging task for the encapsulation of the hydrophilic CQ with hydrophobic PLA NP [42]. Thus, encapsulating CQ with polymeric NP can be a promising approach for increasing the efficiency of CQ in SARS-CoV-2 treatment.

With the conjugation of bio-derived Chitosan nanoparticle with CQ (i.e., CQ-NP) the efficiency of CQ was effectively increased in killing malarial parasites like Plasmodium berghei, in mice as compared to the treatment using free CQ. The parasite loading in the Plasmodium berghei infected mice reduced to $\sim 31 \%$ and $\sim 3 \%$ of the original value on administration of CQ and CQ-NP respectively. This indicates the 10-fold efficacy of CQ-NP as compared to free CQ in killing the malarial parasite. CQ-NP was found to act against the severity of the malarial infection by promoting the production of antioxidant enzymes which helps in mitigating the adverse effects of reactive oxygen species (ROS) enzymes secreted by the malarial parasite. Thus, the action of CQ-NP helps in protecting the host cell from DNA damage [43]

Respiratory viruses like SARS-CoV-2 generate certain enzymes that enhance the ROS enzymes in the infected/morbid regions of the host. Severities of infection by such viruses have been attributed to oxidative stress in the host cells as a result of redox pathway triggered by the action of ROS enzymes secreted by the virus cells [44]. Thus, SARS-CoV-2 viral infection treatment can be potentially improved with CQ while conjugating with chitosan nanoparticle.

\subsection{Reduced Cardiotoxicity Induced by $C Q$}

Both CQ and HCQ along with quinidine and quinine are derivatives of quinoline and have been used against malarial infections caused by the Plasmodium parasites since a long time. However, an older use of quinidine and quinidine-based drugs has been for the treatment of abnormalities in heart rhythm as a Class $1 \mathrm{~A}$ antiarrythmic agent [45]. Quinidine controls the electrical impulses in the heart by blocking sodium and potassium ion channels thus regulating the cardiac electrical activity. This helps in regularizing or slowing the erratic beating of the heart. The adverse effects from the administration of quinidine resulting in prolonged QTc interval have shown development of torsade de pointes which is a life-threatening arrhythmia. Further, co-medication of certain drugs, notably azithromycin has been reported to aggravate the risk of QTc prolongation, thus requiring patients to be closely monitored for cardiac activity in such cases [44]. The action of CQ/HCQ in blocking of potassium ion channels in the cardiac tissue can be considered similar to that of quinine and its stereoisomer quinidine. Thus, this family of drugs can be a cause of potentially lethal arrhythmia if administered without proper medical supervision [46].

The cardiotoxicity arising from chloroquine or quinine family of drugs can be safely bypassed by the use of nano-carriers to deliver the drug at the affected regions of the human body. Size of the nanoparticles is an important advantage and helps in targeted administration to the virus infected cells. There are a wide range of options available amongst nanocarriers ranging from liposomic, micellar to hydrophobic synthetically produced polymers such as PLA, PCL, PEG etc., [42]. The advantage of 
liposomic nano-carriers are their inherent biocompatibility with the human cellular environments, while hydrophobic nature of synthetic polymers such as PLA and PCL help in controlled release of the drugs. Many nano-carriers also have the advantages of being responsive to certain stimuli like temperature, $\mathrm{pH}$, redox environment and thus can be selectively used to release the drug at the intended place of virus infection [38]. Comparative studies on the use of chloroquine phosphate for the treatment of herpes simplex virus type 1 have clearly shown the capability of nanocapsulated CQ in successfully inhibiting $100 \%$ replication of the virus at one-third the concentration of $\mathrm{CQ}$ when administered without nano-carriers. Thus, the use of nano-carriers for chloroquine or quinidine family of drugs if used for the treatment of SARS-CoV-2 infections can be successful in preventing cardiotoxicity mainly due to reduction of overall dosage administered to the patient due to the ability of precision and sustained release of the drug at the site of virus infected cells.

Nanoparticles can also help in reducing drug induced cardiotoxicity for molecules other than quinidine, CQ or HCQ. Halofantrine is an antimalarial drug prescribed to malarial infections resistant to CQ. Halofantrine, like $\mathrm{CQ}$, leads to QTc prolongation and acute cardiotoxicity via potassium channel inhibition. Studies on encapsulation of halofantrine using PLA, PCL and PEG nanocapsules have shown positive response in cardiotoxicity reduction via modification of the drug distribution in the human body. Cardiological alterations were found to be greatly reduced on administration of halofantrine using nanocapsules as compared to that of drug as a solution due to the lower availability of the drug for interaction with the cardiac tissues $[47,48]$.

Quinidine, quinine and CQ are also well known to cause hypotension due to alpha-blocking activity. A high plasma concentration of these drugs after administration, usually through the intravenous or intra-muscular routes is the cause of hypotension during the distribution phase of the drug [49]. Making these drugs available in enclosed or encapsulated form within nano-carriers can safely reduce the plasma concentration of the drug in the distribution phase, thus reducing the risk of hypotension.

\subsection{Reduction of Virus Induced Apoptosis}

Autophagy plays an important role in humans in the regulation of physiological and pathological processes. It is activated for the survival of the cell in some stress environments like starvation, mitochondrial damage, pathological infection etc., [50,51]. Autophagy is a basic mechanism in cells where, damaged unused cellular components are transported via an intermediate organelle, autophagosome to lysosomes for degradation. In the autophagic (Macroautophagy) process, autophagosome and lysosome fuse together and forms autophagic lysosome. This is followed by the degradation of the transported unwanted cellular parts contained in it by lyososomal acid hydrolases [52]. However, cytoplasmic constituents after degradation are again recycled to nutrients for the normal survival of the cells. Though autophagy is of utmost importance for the normal survival of the cells, it supports in the development of some diseases including neurodegeneration, aging, cancer etc.

In cancer, in the initial stage of tumor cell growth, autophagic mechanism is upregulated and prevents the initiation of the tumor formation. While after tumor formation, autophagy supports its growth, metabolism and progression, and helps the cancer cells to overcome the intracellular environmental stress [53]. It is believed that autophagy inhibits apoptosis (programmed cell death) of the cancer cells thus protecting the tumor cells [54]. Anticancer drugs such as CQ kills the cancer cells by inhibiting autophagy and promoting apoptosis of the cancer infected cells. However, in the procedure of drug application, autophagy develops acquired resistance by preventing any anticancer drug from killing/terminating the cancer cells. Some patients may show a declined response to CQ due to acquired resistance to the drug when used for cancer therapy after some time of application. Nanoparticle was effectively used to overcome this acquired resistance shown by cancer cells. The apoptosis of the QGY cancer cell has been significantly enhanced by the use of chitosan-nanoparticle-encapsulated CQ as compared to the free-CQ used for treatment [55]. This results in the inhibition of autophagy by blocking autophagosome fusion and degradation. This is how the nanoparticle encapsulated CQ helps in overcoming the acquired resistance shown by the cells. Similarly, drug resistance shown by H1N1 virus to the drug Amantadine (AM) is overcome by the conjugation of Amantadine with silver nanoparticle (Ag NPs) [56]. It was observed that with the conjugation of Ag NPs, there was an enhancement of cellular uptake of the drug which blocked the H1N1 viral infection by inhibiting viral proteins Hemagglutinin (HA) and Neuraminidase (NA). The virus induced apoptosis was significantly reversed with the co-delivery of Ag NPs and thereby overcoming against the virus induced drug resistance. Similar study was carried out by using Selenium nanoparticles against H1N1 virus treatment with Ribavirin drug [57].
As scientists carry out across the age-group clinical trials of CQ/HCQ to counter the spread of SARS-CoV-2, it has become evident that the virus has undergone rapid transformations with over 200 natural mutations recorded worldwide [58]. The new strains of virus may induce drug resistance to CQ and HCQ [59]. Nanotechnology can be the answer to the possible drug resistance offered by the mutant strains of the virus. Nanoparticle conjugated drugs are also expected to successfully deliver the drug to the virus infected cells.

\section{CQ Inhibits Nanoparticles}

Though different nano-platforms are found to enhance drug efficiency in the treatment of different diseases including cancer and viral treatments, it is reported that above $90 \%$ of the therapeutic nanoparticles used, gets deposited in the liver and spleen via endocytosis by the action of resident macrophages in liver [60]. Macrophages in the liver engulf foreign objects that do not have the type of proteins specific to healthy body cells on its surface. CQ works as an efficient inhibitor of nanoparticle deposition in the liver and spleen cells of mice by suppressing endocytosis [61]. In the early stages of endocytosis, CQ changes the level of proteins specially, phosphatidylinositol binding clathrin assembly protein (PICALM) which are involved in the endocytosis process of the liver cells. As PICALM plays an important role in clathrin-dependent endocytosis, suppression of PICALM results in the inhibition of clathrin-dependent endocytosis process of the NPs [62]. As the final step of the inhibition of nanoparticle deposition in the liver, $\mathrm{CQ}$ induces dysfunction of the lysosomes by increasing its $\mathrm{pH}$. These lysosomes would have otherwise acted as the end compartment for internalisation of the nanoparticles in the endocytosis process [63]. Thus, CQ in combination with nanoparticles can be used as a therapeutic medicine against SARS-CoV-2 and at the same time suppress the deposition of nanoparticles by acting as macrophagemodulator with respect to the endocytosis of nanoparticles in the liver and spleen.

\section{Conclusions}

CQ and HCQ are the key drugs being investigated for the fight against the SARS-CoV-2. CQ acts by preventing the attachment of the virion particle onto the host cell through sialic acid present in the virus. The challenges of using $\mathrm{CQ}$ is mostly due to the administration of high dosage such as cardiotoxicity via QTc prolongation, decreased drug efficiency and development of acquired drug resistance by the virus and can be potentially overcome by the use of nano-carriers as a drug delivery vehicle. Nano-carriers have the unique advantages of keeping the CQ dosage intact and delivering the medicine at a programmed rate of controlled diffusion at the sites of virus infection. There have been examples of successful use of nanotechnology for drug delivery such as CQ encapsulated with PLA nanoparticles against herpes simplex virus, silver nanoparticle-Amantadine conjugated systems against H1N1 virus infection, and chitosan nanoparticle encapsulated CQ against Plasmodium berghei parasite. The authors would strongly recommend further experimental investigation on the use of $\mathrm{CQ} / \mathrm{HCQ}$ with nano-carriers so that the disadvantages of using CQ arising out of possible high dosage due to acquired drug resistance can be offset by the benefits of using nanocarriers for the drug. The ability of CQ in inhibiting the deposition of nanoparticles by mechanisms such as suppression of endocytosis and internalization in the liver and spleen further makes for a strong proposition for the CQ-NP combination in the fight against SARS-CoV-2.

\section{References}

[1] P. Zhou, X.L. Yang, X.G. Wang, B. Hu, L. Zhang, et al., A pneumonia outbreak associated with a new coronavirus of probable bat origin, Nature 579 (2020) 270-273.

[2] https://www.who.int/emergencies/diseases/novel-coronavirus2019/events-as-they-happen (Accessed on: 25.03.2020)

[3] T.G. Ksiazek, D. Erdman, C.S. Goldsmith, S.R. Zaki, T. Peret, et al., SARS Working Group, A novel coronavirus associated with severe acute respiratory syndrome, N. Engl. J. Med. 348 (2003) 1953-1966

[4] Y. Chen, Q. Liu, D. Guo, Emerging coronaviruses: Genome structure, replication and pathogenesis, J. Med. Virol. 92 (2020) 418-423.

[5] M. Tobaiqy, M. Qashqary, S. Al-Dahery, A. Mujallad, A.A. Hershan, M.A. Kamal N. Helmi, Therapeutic management of patients with COVID-19: a systematic review, Infect. Prevent. Prac. 2 (2020) 100061:1-21.

[6] P. Colson, J.M. Rolain, D. Raoult, Chloroquine for the 2019 novel coronavirus SARS-CoV-2, Int. J. Antimicrob. Agents 55 (2020) 105923:1-3.

[7] A. Savarino, L. D. Trani, I. Donatelli, R. Cauda, A. Cassone, New insights into the antiviral effects of chloroquine, Lancet. Infect. dis. 6 (2006) 67-69. 
[8] J.M. Rolain, P. Colson, D. Raoult, Recycling of chloroquine and its hydroxyl analogue to face bacterial, fungal and viral infections in the 21st century, Int. J. Antimicrob. Agents 30 (2007) 297-308.

[9] R. Sarma, D. Mohanta, Luminescence and bio-imaging response of thio-glycolic acid (TGA) and sodium dodecyl sulfate (SDS)-coated fluorescent cadmium selenide quantum dots, J. Lumin. 161 (2015) 395-402.

[10] J.U. Menon, P. Jadeja, P. Tambe, K. Vu, B. Yuan, K.T. Nguyen, Nanomaterials for photo-based diagnostic and therapeutic applications, Theranostics 3 (2013) 152-166.

[11] N. Zhu, D. Zhang, W. Wang, X. Li, B. Yang, et al., A novel coronavirus from patients with pneumonia in China, 2019, N. Engl. J. Med. 382 (2020) 727-733.

[12] C. Traving, R. Schauer, Structure, function and metabolism of sialic acids, Cell. Mol. Life Sci. 54 (1998) 1330-1349.

[13] S. Hinderlich, W. Weidemann, T. Yardeni, R. Horstkorte, M. Huizing, UDPGlcNAc 2-Epimerase/ManNAc Kinase (GNE): A master regulator of sialic acid synthesis, Top Curr. Chem. 366 (2015) 97-137.

[14] F. Li, W. Li, M. Farzan, S.C. Harrison, Structure of SARS coronavirus spike receptor-binding domain complexed with receptor, Science 309 (2005) 18641868.

[15] I. Hamming, W. Timens, M.L.C. Bulthuis, A.T. Lely, G.J. Navis, H.V. Goor, Tissue distribution of ACE2 protein, the functional receptor for SARS coronavirus. A first step in understanding SARS pathogenesis, J. Pathol 203 (2004) 631-637.

[16] M. Hoffmann, H.K. Weber, S. Schroeder, N. Krüger, T. Herrler, et al., SARS-CoV2 cell entry depends on ACE2 and TMPRSS2 and is blocked by a clinically proven protease inhibitor, Cell 181 (2020) 271-280.

[17] M.J. Vincent, E. Bergeron, S. Benjannet, B.R. Erickson, P.E. Rollin, et al., Chloroquine is a potent inhibitor of SARS coronavirus infection and spread, Virol. J. 2 (2005) 69:1-10.

[18] C.A. Devaux, J. Rolain, P. Colson, D. Raoult, New insights on the antiviral effects of chloroquine against coronavirus: what to expect for COVID-19?, Int. J. Antimicrob. Agents 55 (2020) 105938:1-6.

[19] D.J. Browning, Pharmacology of chloroquine and hydroxychloroquine, in: D.J. Browning hydroxychloroquine and chloroquine retinopathy, Springer, New York, 2014, pp.35-63.

[20] M.A.A. Al-Bari, Chloroquine analogues in drug discovery: new directions of uses, mechanisms of actions and toxic manifestations from malaria to multifarious diseases, J. Antimicrob. Chemother. 70 (2015) 1608-1621.

[21] R.E. Martin, R.V. Marchetti, A.I. Cowan, S.M. Howitt, S. Bröer, K. Kirk, Chloroquine transport via the malaria parasite's chloroquine resistance transporter, Science 325 (2009) 1680-1682.

[22] Here's how nanomedicine provides insights into chloroquine's efficacy against COVID-19? Nano Science, Technology and Industry Scoreboardhttps://statnano.com/news/67552/Here\%E2\%80\%99s-HowNanomedicine-Provides-Insights-into-Chloroquine\%E2\%80\%99s-EfficacyAgainst-COVID-19, 2020. (Accessed on: 12.07.2020)

[23] A. Savarino, J.R. Boelaert, A. Cassone, G. Majori, R. Cauda, Effects of chloroquine on viral infections: an old drug against today's diseases, Lancet Infec. Dis. 3 (2003) 722-727.

[24] B. Russell, C. Moss, G. George, A. Santaolalla, A. Cope, S. Papa, M.V. Hemelrijck, Associations between immune-suppressive and stimulating drugs and novel COVID-19-a systematic review of current evidence, Ecanc. Med. Sci. 14 (2020) 1022:1-43.

[25] K.S. Joshy, S. Snigdha A. George, N. Kalarikkal, A. Laly. Pothen, S. Thomas, Poly (vinyl pyrrolidone)-lipid based hybrid nanoparticles for anti viral drug delivery, Chem. Phys. Lipids 210 (2018) 82-89.

[26] S.S. Bawage, P.M. Tiwari, A. Singh, S. Dixit, S.R. Pillai, V.A. Dennis, S.R. Singh, Gold nanorods inhibit respiratory syncytial virus by stimulating the innate immune response, Nanomed-nanotechnol. 12 (2016) 2299-2310.

[27] Y. Shen, J. Tu, Preparation and ocular pharmacokinetics of ganciclovir liposomes, AAPS J. 9 (2007) E371-E377.

[28] R.A. Petros, J.M. DeSimone, Strategies in the design of nanoparticles for therapeutic applications, Nat. Rev. Drug Discov. 9 (2010) 615-627.

[29] S.D. Mahajan, Anti-HIV-1 nanotherapeutics: promises and challenges for the future, Int. J. Nanomed. 7 (2012) 5301-5314

[30] G.V. Patel, V.B. Patel, A. Pathak, S.J. Rajput, Nanosuspension of efavirenz for improved oral bioavailability: formulation optimization, in vitro, in situ and in vivo evaluation, Drug Dev. Ind. Pharm 40 (2014) 80-91.

[31] J. Torrecilla, A. Rodríguez-Gascón, M.A. Solinís, A. Rodríguez, Lipid nanoparticles as carriers for RNAi against viral infections: current status and future perspectives, BioMed Res. Int. 2014 (2014) 161794:1-18.

[32] F.D. Cojocaru, D. Botezat, I. Gardikiotis, C.M. Uritu, G. Dodi, et al., Nanomaterials designed for antiviral drug delivery transport across biological barriers, Pharmaceutics 12 (2020) 171:1-34.

[33] D. Lembo, M. Donalisio, A. Civra, M. Argenziano, R. Cavalli, Nanomedicine formulations for the delivery of antiviral drugs: a promising solution for the treatment of viral infections, Expert opin. drug deliv. 15 (2018) 93-114.

[34] R. Mainardes, C. Diedrich, The potential role of nanomedicine on COVID-19 therapeutics, Ther. Deliv. 2020, https://www.futurescience.com/doi/10.4155/tde-2020-0069. (Accessed on: 11.07.2020)
[35] G. Manic, F. Obrist, G. Kroemer, I. Vitale, L. Galluzzi, Chloroquine and hydroxychloroquine for cancer therapy, Mol. Cell. Oncol 1 (2014) e299111e2991111.

[36] A. Bjelle, A. Björnham, A. Larsen, T. Mjörndal, Chloroquine in long-term treatment of rheumatoid arthritis, Clin. Rheumatol. 2 (1983) 393-399.

[37] J. Kearney, Chloroquine as a potential treatment and prevention measure for the 2019 novel coronavirus: A Review, Preprints (2020) 2020030275:1-24.

[38] R. Cheng, F. Meng, C. Deng, H. AntonKlok, Z. Zhong, Dual and multi-stimul responsive polymeric nanoparticles for programmed site-specific drug delivery, Biomaterials 34 (2013) 3647-3657.

[39] H.A. Santos, L.M. Bimbo, L. Peltonen, J. Hirvonen, Inorganic nanoparticles in targeted drug delivery and imaging, in: P.V. Devarajan and S. Jain (Eds.) Targeted drug delivery : Concepts and design, Springer International Publishing, Cham, 2015, pp. 571-613.

[40] D. Bennet, S. Kim, Polymer nanoparticles for smart drug delivery, in: A.D. Sezer (Ed.), Application of nanotechnology in drug delivery, InTech, Rijeka, 2014, p.257.

[41] J.K. Patra, G. Das, L.F. Fraceto, E.V.R. Campos, Md.P.R. Torres, et al., Nano based drug delivery systems: recent developments and future prospects, J. Nanobiotech 16 (2018) 71:1-33.

[42] T.L.C. Lima, R.C. Feitosa, Ed. Santos-Silva, A.Md. Santos-Silva, E.Md.S Siqueira, et al., Improving encapsulation of hydrophilic chloroquine diphosphate into biodegradable nanoparticles: A promising approach against herpes virus simplex-1 infection, Pharmaceutics 10 (2018) 255:1-18.

[43] Nanochloroquine for drug-resistant malaria parasite, Nature India, 2013, https://www.natureasia.com/en/nindia/article/10.1038/nindia.2013.155 (Accessed on: 02.04.2020)

[44] O.A. Khomich, S.N. Kochetkov, B. Bartosch, A.V. Ivanov, Redox biology of respiratory viral infections, Viruses 10 (2018) 392:1-27.

[45] J.K. Aronson, Meyler's side effects of drugs, Sixteenth Edn., Elsevier, Oxford, London, 2016, pp.17-26.

[46] T.J. Bauman, Chloroquine and hydroxychloroquine in the era os SARSCoV2 caution on their cardiac toxicity, Pharmacother. 40 (2020) 387-388.

[47] E.A. Leite, A.G. Guimarães, H.N. Guimarães, G.L.L. Machado-Coelho, G. Barratt, V.C.F. Mosqueira, Cardiotoxicity reduction induced by halofantrine entrapped in nanocapsule devices, Life Sci. 80 (2007) 1327-1334.

[48] A.M. Grumezescu, Antimicrobial nanoarchitectonics: From synthesis to applications, First Edn., Elsevier Science, Amsterdam, Netherlands, 2017.

[49] N.J. White, Cardiotoxicity of antimalarial drugs, Lancet Inf. Dis. 7 (2007) 549 558.

[50] H. Vakifahmetoglu-Norberg, H.g. Xia, J. Yuan, Pharmacologic agents targeting autophagy. J. Clin. Invest. 125 (2015) 5-13.

[51] G. Hewitt, V.I. Korolchuk, Repair, reuse, recycle: The expanding role of autophagy in genome maintenance, Trends. Cell Biol. 27 (2017) 340-351.

[52] Y. Chun, J. Kim, Autophagy: An essential degradation program for cellular homeostasis and life, Cells 7 (2018) 278:1-26.

[53] V. Desantis, I. Saltarella, A. Lamanuzzi, M.A. Mariggiò, V. Racanelli, A. Vacca, M.A. Frassanito, Autophagy: A new mechanism of prosurvival and drug resistance in multiple myeloma, Transl. Oncol. 11 (2018) 1350-1357.

[54] H. Zhang, Z. Chen, Autophagy and cell death: Antitumor drugs targeting autophagy, in: H.G. Muhtasib, O.N. Rahal (Ed.), Programmed cell death, Intech Open, London, UK, 2019.

[55] L. Zhao, G. Yang, Y. Shi, C. Su, J. Chang, Co-delivery of Gefitinib and chloroquine by chitosan nanoparticles for overcoming the drug acquired resistance, J. Nanobiotech. 13 (2015) 57:1-10.

[56] Y. Li, Z. Lin, M. Zhao, M. Guo, T. Xu, et al., The reversal of H1N1 influenza virusinduced apoptosis by silver nanoparticles functionalized with Amantadine, RSC Adv. 6 (2016) 89679-89686.

[57] Z. Lin, Y. Li, G. Gong, Y. Xia, C. Wang, et al., Restriction of H1N1 influenza virus infection by selenium nanoparticles loaded with ribavirin via resisting caspase-3 apoptotic pathway, Int. J. Nanomed. 13 (2018) 5787-5797.

[58] https://www.theweek.in/news/sci-tech/2020/05/08/coronavirus-ismutating-and-one-particular-strain-is-worrying-researchers.html (Accessed on: 20.05.2020)

[59] A.K. Singh, A. Singh, A. Shaikh, R. Singh, A. Misra, Chloroquine and hydroxychloroquine in the treatment of COVID-19 with or without diabetes: A systematic search and a narrative review with a special reference to India and other developing countries, Diabetes Metab. Syndr. 14 (2020) 241-246.

[60] J. Wolfram, S. Nizzero, H. Liu, F. Li, G. Zhang, et al., A chloroquine-induced macrophage-preconditioning strategy for improved nanodelivery, Sci. Rep. 7 (2017) 13738:1-13.

[61] J. Pelt, S. Busatto, M. Ferrari, E.A. Thompson, K. Mody, J. Wolfram, Chloroquine and nanoparticle drug delivery: A promising combination, Pharmacol. Therap. 191 (2018) 43-49.

[62] T.Y. Hu, M. Frieman, J. Wolfram, Insights from nanomedicine into chloroquine efficacy against COVID-19, Nat. Nanotech. 15 (2020) 247-249.

[63] G. Sahay, D.Y. Alakhova, A.V. Kabanov, Endocytosis of nanomedicines, J. Control Release 145 (2010) 182-195. 